\title{
Cohort Analysis of Exacerbation Rates in Adolescent and Adult Patients Initiating Inhaled Corticosteroids for Asthma: Different Dose-Response Profile by Particle Size
}

Dirkje S. Postma · Alan Kaplan · Joan B. Soriano · Jonathon Grigg •

Theresa W. Guilbert · Wim van Aalderen · Nicolas Roche •

Anne Burden · Elizabeth V. Hillyer · Elliot Israel · David B. Price

Received: December 29, 2016 / Published online: April 12, 2017

(C) The Author(s) 2017. This article is an open access publication

\section{ABSTRACT}

Introduction: Targeting small airway inflammation could lead to improved clinical outcomes in asthma. Previous observational studies concluded that therapy with extrafine-particle [mass median aerodynamic diameter (MMAD)

Enhanced content To view enhanced content for this article go to www.medengine.com/Redeem/ F8F7F0601CCF240D.

Electronic supplementary material The online version of this article (doi:10.1007/s41030-017-0037-3) contains supplementary material, which is available to authorized users.

\section{S. Postma}

Department of Pulmonary Medicine, University of Groningen, University Medical Center Groningen, Groningen, Netherlands

\section{A. Kaplan}

Family Physician Airways Group of Canada,

Richmond Hill, ON, Canada

J. B. Soriano - A. Burden - E. V. Hillyer - D. B. Price Observational and Pragmatic Research Institute, Singapore, Singapore

\section{J. B. Soriano}

Instituto de Investigación Hospital Universitario de La Princesa (IISP), Universidad Autónoma de

Madrid, Cátedra UAM-Linde, Madrid, Spain

J. Grigg

Blizard Institute, Queen Mary University London, London, UK
$<2 \mu \mathrm{m}]$ inhaled corticosteroids (ICS) is associated with similar or better odds of achieving asthma control at lower prescribed doses than fine-particle ICS (MMAD $=2-5 \mu \mathrm{m})$. However, while it is believed that the dose-response for ICS reaches a plateau at sub-maximal doses, it is not clear whether such a plateau occurs with extrafine-particle ICS.

Methods: To evaluate the potential effect of ICS particle size on dose-response of asthma-related outcomes, we studied severe exacerbation rates in a historical patient cohort of UK adults and adolescents with asthma initiating extrafine- or fine-particle ICS. We extracted electronic medical record data centralized at primary care

T. W. Guilbert

Cincinnati Children's Hospital and Medical Center,

Cincinnati, $\mathrm{OH}, \mathrm{USA}$

W. van Aalderen

Emma Children's Hospital AMC, Amsterdam, Netherlands

\section{N. Roche}

Cochin Hospital Group (AP-HP), University Paris

Descartes (EA2511), Paris, France

E. Israel

Brigham and Women's Hospital and Harvard Medical School, Boston, MA, USA

D. B. Price $(\square)$

Centre of Academic Primary Care, University of

Aberdeen, Polwarth Building, Foresterhill,

Aberdeen AB25 2ZD, UK

e-mail: dprice@opri.sg 
practices, where information from secondary care and hospitalizations is also aggregated. Data were collected over one baseline (pre-initiation) and one outcome year.

Results: Of 32,235 patients with asthma (aged 12-80 years), 54\% initiated ICS with extrafine-particle ICS and $46 \%$ with fine-particle ICS. Overall, mean age (SD) was 41 (17) years; $60 \%$ female; $24 \%$ current and 20\% ex-smokers. We found a greater $(P<0.001)$ reduction in exacerbation rate at higher as compared with lower doses of extrafine-particle ICS: for patients initiating on $\leq 125 \mu \mathrm{g} /$ day the reduction was $-0.016(95 \% \mathrm{CI}-0.038,0.006)$ exacerbations per year and for those initiating on $>250 \mu \mathrm{g} /$ day the reduction was $-0.072(-0.095$, $-0.049)$. No significant dose-response relationship was observed for patients initiating on fine-particle ICS [reduction in exacerbations per year: $-0.041(-0.070,-0.012)$ at the lowest doses and $0.009(-0.017,0.036)$ at the highest, $P=0.856$, despite similar exacerbation rates in the baseline period for both cohorts $(P=0.40)]$. Conclusion: Our findings suggest that extrafine-particle ICS is associated with a reduction in exacerbation rates in a dose-dependent fashion in an adult asthma population. This dose-response relationship was not observed with fine-particle ICS.

ENCePP Trial Registration: EUPAS8840.

Keywords: Dose response; Particle size; Real-life research; Beclomethasone dipropionate; Fluticasone propionate

\section{INTRODUCTION}

Asthma is a heterogeneous disease characterized by chronic inflammation and remodeling of the airways. There is compelling evidence that both central and peripheral airway inflammation are involved in this disease. Peripheral airways are defined as those that have an internal diameter of $<2 \mathrm{~mm}$, do not contain cartilage in their walls, and extend to the alveolar ducts. Peripheral lung inflammation has been found in patients with mild asthma and has been suggested to play a part in the clinical expression and worse control of asthma [1]. The outer wall of peripheral airways has recently been recognized as the major site of remodeling in patients with fatal asthma [2]. Moreover, early closure of peripheral airways has been shown to characterize patients with "difficult to control" asthma [3].

International guidelines affirm inhaled corticosteroids (ICS) as the mainstay of pharmacological asthma treatment [4], as they reduce eosinophilic inflammation, improve pulmonary function, and lead to reduction in symptoms and exacerbations. Studies examining fine-particle ICS have indicated a relatively flat dose-response of ICS with respect to clinical outcomes, including exacerbations $[5,6]$. Most of the therapeutic benefits of ICS in patients with mild to moderate asthma are achieved at relatively low doses, equivalent to $100-250 \mu \mathrm{g}$ fluticasone propionate per day, with a maximum achievable benefit at around $500 \mu \mathrm{g} /$ day $[6,7]$. Randomized controlled trials (RCTs) have found that low dose ICS is as effective as high dose ICS at achieving asthma control [8]. However, lack of data limits these findings due to paucity of dose response studies. Since fine-particle ICS effectively reduce inflammation in the larger airways, but may not reach the peripheral airways, this plateau in dose-response might be due, at least partially, to residual inflammation in the small airways [9]. Inflammatory and structural changes occur throughout the airways. Therefore, anti-inflammatory treatment should be targeted at both central and peripheral airways to achieve inflammation suppression throughout the bronchial tree.

The effect of inhalation therapy is determined by multiple factors, including the amount of therapy reaching the lungs and its distribution throughout the airways [10]. Targeting inhaled drug formulations to the peripheral airways is not efficient, with most of the formulations being deposited in the central airways. A way to overcome this is to reduce the mean particle size of the inhaled drug to a mass median aerodynamic diameter (MMAD) of $<2 \mu \mathrm{m}$. As particle size decreases, the fraction reaching the peripheral areas of the lung increases [11]. Drug formulations that have a small particle size are called extrafine, and RCTs have shown that, when prescribed as a single 
therapy, extrafine ICS provide additional clinical benefits compared with the same fine-particle therapy or placebo [12, 13]. Extra-fine-particle dose-response may continue beyond that of fine-particle ICS therapy owing to greater peripheral lung deposition [14]. This hypothesis is supported by real-life research reporting similar, or in some instances significantly better, asthma control outcomes with extrafine drug formulations compared with fine-particle ones, and at a lower dose $[15,16]$. Using nationwide databases to conduct real-life studies allows the generation of large and heterogeneous populations of patients with asthma. This permits us to examine longer-term outcomes, providing information to complement the results of RCTs through the inclusion of unselected representative patients managed in real-life clinical practice [17-19]. Thus, exploring the dose-response relationship of extrafine versus fine-particle ICS may have clinical implications but may also provide insights on the clinical relevance of the "distal airways inflammation" concept. The aim of this study was to investigate the hypothesis that the dose-response effect on the change in the rate of severe asthma exacerbations is steeper for extrafine particle ICS than for fine-particle ICS in a real-life dataset.

\section{METHODS}

\section{Study Design}

This historical, within-treatment cohort analysis consisted of a 1-year baseline period for patient characterization, followed by a 1-year outcome evaluation period. The baseline and outcome periods were separated by the index date. The index date was defined as when patients received their first prescription of ICS as either extrafine-particle ICS [hydrofluoroalkane beclomethasone diproprionate (EF HFA-BDP; Qvar $^{\circledR}$ ) or ciclesonide $\left(\right.$ Alvesco $\left.^{\circledR}\right)$ ] or fine-particle ICS [fluticasone propionate (FP) or fine-particle beclomethasone dipropionate (BDP) $\left(\right.$ Clenil $\left.\left.^{\circledR}\right)\right]$.

The study protocol was designed prior to data extraction by an independent steering committee and registered with the European Network of Centers for Pharmacoepidemiology and Pharmacovigilance (trial registration number EUPAS8840).

\section{Data Source}

The study utilized two large UK primary care databases, the Optimum Patient Care Research Database (OPCRD) [20] and the Clinical Practice Research Datalink (CPRD) [21]. At the time of the study, the OPCRD contained anonymous, longitudinal data for over 84,534 patients with asthma. The OPCRD is approved by the Health Research Authority of the UK NHS for clinical research use (REC reference: 15/EM/0150). The CPRD is the world's largest validated computerized database of anonymized longitudinal medical records for primary care [22]. At the time of the study, data were available for approximately 13 million patient records from over 450 primary care practices throughout the UK. Records contain complete prescribing and coded diagnostic and clinical information as well as information on tests requested, laboratory results and referrals made at, or following on from, each consultation [23]. This was a retrospective study utilizing anonymized patient data, for which formal consent is not required.

\section{Patients}

Eligible patients were aged 12 to 80 years with mild to moderate asthma, received $\geq 2$ asthma prescriptions during the outcome year (i.e., $\geq 1$ in addition to the ICS prescription at the index date), initiated ICS as a single therapy, and had 2 years of continuous practice data (comprising $\geq 1$ year of baseline data and 1 year of outcome data). Patients were excluded if they had a diagnosis for any chronic respiratory disease other than asthma at any time in their records, received maintenance oral corticosteroids during the baseline year, received a co-prescription for a long-acting beta-agonist (LABA), leukotriene receptor antagonist (LTRA), or theophylline, and were a smoker or ex-smoker over the age of 60 (to minimize the risk of including patients with COPD). 


\section{Outcome Measures}

The primary endpoint was severe asthma exacerbation rate. We evaluated outcomes for three ICS dose categories ( $\leq 125 \mu \mathrm{g} /$ day, $125-$ $250 \mu \mathrm{g} /$ day, $>250 \mu \mathrm{g} /$ day FP equivalent) in each arm (fine and extrafine particles). We plotted the absolute change in severe exacerbations from baseline to outcome. We defined a severe exacerbation as an asthma-related hospital admission, emergency department attendance, or an acute course of oral corticosteroids (based on the American Thoracic Society/European Respiratory Society definition) [24]. Asthma treatment guidelines recommend that extrafine-particle ICS [i.e., ciclesonide $\left(\right.$ Alvesco ${ }^{\circledR}$ ) and extrafine-particle hydrofluoroalkane beclomethasone dipropionate (EF HFA-BDP; Qvar $^{\circledR}$ )] are prescribed at the same dose as fine-particle fluticasone propionate (FP) (e.g. $100 \mu \mathrm{g}=100 \mu \mathrm{g}$ ), and at half the dose of fine-particle beclomethasone suspension $(100 \mu \mathrm{g} \mathrm{BDP}=50 \mu \mathrm{g}$ FP) [25].

\section{Statistical Analysis}

Summary statistics for all baseline and outcome variables were generated to characterize patients in each ICS dose category. We used the Mann-Whitney test for continuous variables and the Chi square test for categorical variables to compare treatment groups at baseline; and the Kruskal-Wallis Test to identify the differences in exacerbation rates by dose. Doses were expressed as FP equivalent. Statistically significant results were defined as $P<0.05$. The mean and $95 \%$ confidence interval of the change in exacerbation rate from baseline to outcome for three ICS dose categories was plotted.

No prospective power calculation was carried out since the sample size was determined by the number of eligible patients in the OPCRD per the inclusion criteria.

All analyses were carried out using IBM SPSS Statistics version 21 (IBM SPSS Statistics, Feltham, Middlesex, UK), SAS version 9.3 (SAS Institute, Marlow, Buckinghamshire, UK), and Microsoft Office Excel 2013 (Microsoft Corp., Redmond, Washington, USA).

\section{RESULTS}

\section{Patients}

We identified 508,349 patients of whom 32,235 met the study eligibility criteria (Figure S1, online supplement). Of these, 17,023 (53\%) were prescribed EF HFA-BDP $\left(\right.$ Qvar $\left.^{\circledR}\right), 247$ $(0.8 \%)$ ciclesonide $\left(\right.$ Alvesco $\left.^{\circledR}\right), 4693$ (15\%) FP via pMDI, 3046 (9\%) FP via DPI, and 7226 (22\%) fine-particle beclomethasone dipropionate $\left(\right.$ Clenil $\left.{ }^{\circledR}\right)$.

The mean age (SD) of the total population was 41 (17) years, 60\% were female, and $44 \%$ were current or ex-smokers (under the age of $60)$. Baseline exacerbation rates were comparable for patients initiating on extrafine- and fine-particle ICS $(P=0.40)$ (Table 1$)$. Peak expiratory flow was also similar between patients initiating on fine or extrafine $(P=0.309$; Table 1). The higher dose categories had greater proportions of patients with 1 or $>2$ baseline exacerbations, compared with the lowest dose category, which was consistent across both the extrafine- and fine-particle groups (Table 2).

\section{Outcomes}

Using the Kruskal-Wallis test we found there was a significant difference in the primary study endpoint, rate of severe exacerbations, between the dose categories of ICS for both extrafineand fine-particle cohorts (Fig. 1). There was a greater $(P<0.001)$ reduction in exacerbation rate at higher doses of extrafine-particle ICS compared with lower and medium doses (Fig. 1; Table 3). Specifically, for patients initiating on $\leq 125 \mu \mathrm{g}$ /day the reduction was -0.016 (95\% CI, $-0.038,0.006)$ exacerbations per year. For those initiating on $>125-250 \mu \mathrm{g} /$ day of extrafine-particle ICS, the reduction was -0.423 (95\% CI, $-0.057,-0.028)$ and for those initiating on $>250 \mu \mathrm{g} /$ day the reduction was -0.072 (95\% CI, $-0.095,-0.049)$. An increasing dose-response relationship was not observed for patients initiating on fine-particle ICS. There were significant reductions in exacerbations per year in the lowest dose category $[-0.041$ (95\% CI, -0.070 , $-0.012)]$, and the medium dose category 
Table 1 Demographic and clinical characteristics of patients with asthma at baseline year

\begin{tabular}{|c|c|c|c|c|}
\hline Characteristic & $\begin{array}{l}\text { Extrafine-particle } \\
\text { ICS } \\
(n=17,270)\end{array}$ & $\begin{array}{l}\text { Fine-particle } \\
\text { ICS } \\
(n=14,965)\end{array}$ & $\begin{array}{l}\text { Total } \\
(n=32,235)\end{array}$ & $P$ value \\
\hline Age (years), mean (SD) & $41.5(16.4)$ & $39.9(17.0)$ & $40.8(16.7)$ & $<0.001^{*}$ \\
\hline Female, $n(\%)$ & $10,395(60.2)$ & $9055(60.5)$ & $19,450(60.3)$ & $0.562^{¥}$ \\
\hline \multicolumn{5}{|l|}{ Recorded smoking status, $n(\%)$} \\
\hline Non-smoker & $9046(52.4)$ & $8471(56.6)$ & $17,517(54.3)$ & \\
\hline Current smoker & $4303(24.9)$ & $3329(22.2)$ & $7632(23.7)$ & $<0.001^{¥}$ \\
\hline Ex-smoker & 3577 (20.7) & 2835 (18.9) & $6412(19.9)$ & \\
\hline Unknown & $344(2.0)$ & $330(2.2)$ & $674(2.1)$ & \\
\hline $\begin{array}{l}\text { Percent predicted peak flow readings (\%), mean } \\
\quad(\mathrm{SD})\end{array}$ & $83.58(19.01)$ & $83.29(19.70)$ & $83.45(19.33)$ & $0.309^{*}$ \\
\hline$N$ (\% non-missing) & $11,863(63.8)$ & $11,175(58.1)$ & $23,038(60.9)$ & \\
\hline Year of first ICS prescription, median (IQR) & $2005(2003,2007)$ & $\begin{array}{l}2007(2001 \\
2008)\end{array}$ & $\begin{array}{l}2006(2002 \\
2008)\end{array}$ & $<0.001^{¥}$ \\
\hline ICS dose $(\mu \mathrm{g})$, mean $(\mathrm{SD})$ & $238.1(116.7)$ & $343.0(288.9)$ & $286.8(220.8)$ & $<0.001^{*}$ \\
\hline \multicolumn{5}{|c|}{ Severe exacerbations during baseline year (ATS/ERS defined), $n(\%)$} \\
\hline 0 & $14,182(82.1)$ & $12,248(81.8)$ & $26,430(82.0)$ & \\
\hline 1 & $2518(14.6)$ & $2149(14.4)$ & $4667(14.5)$ & $0.052^{¥}$ \\
\hline$\geq 2$ & $570(3.3)$ & $568(3.8)$ & $1138(3.5)$ & \\
\hline \multicolumn{5}{|l|}{ Average SABA daily dose $(\mu \mathrm{g})$} \\
\hline None, $n(\%)$ & $7533(43.6)$ & $6499(43.4)$ & $14,032(43.5)$ & \\
\hline$\leq 100, n(\%)$ & $4332(25.1)$ & $3495(23.4)$ & $7827(24.3)$ & $<0.001^{\ddagger}$ \\
\hline $101-200, n(\%)$ & $2951(17.1)$ & $2618(17.5)$ & $5569(17.3)$ & \\
\hline$>200, n(\%)$ & $2454(14.2)$ & $2353(15.7)$ & $4807(14.9)$ & \\
\hline Risk domain asthma control, $n(\%)$ & $11,801(68.3)$ & $10,405(69.5)$ & $22,206(68.9)$ & $0.021^{¥}$ \\
\hline GERD diagnosis or therapy, $n(\%)$ & $3607(20.9)$ & $3183(21.3)$ & $6790(21.2)$ & $0.399^{¥}$ \\
\hline IHD diagnosis, $n$ (\%) & $777(4.5)$ & $545(3.6)$ & $1322(4.1)$ & $<0.001^{¥}$ \\
\hline Rhinitis diagnosis or therapy, $n(\%)$ & $5016(29.0)$ & $4830(32.3)$ & $9846(30.5)$ & $0.011^{¥}$ \\
\hline
\end{tabular}

$S D$ standard deviation, GERD gastroesophageal reflux disease, $I H D$ ischemic heart disease, $S A B A$ short-acting beta agonist, ICS inhaled corticosteroid, LABA long-acting beta agonist

* Mann-Whitney test

$¥$ Chi squared test 
Table 2 Baseline characteristics for patients initiated on different doses of extrafine- and fine-particle ICS

\begin{tabular}{|c|c|c|c|c|c|c|c|c|}
\hline \multirow[b]{2}{*}{ Characteristic } & \multicolumn{4}{|c|}{$\begin{array}{l}\text { Extrafine-particle ICS daily } \\
\text { dose at initiation }^{\mathrm{a}}(\mu \mathrm{g})\end{array}$} & \multicolumn{4}{|c|}{$\begin{array}{l}\text { Fine-particle ICS daily } \\
\text { dose at initiation }^{\mathrm{a}}(\mu \mathrm{g})\end{array}$} \\
\hline & $>0-125$ & $>125-250$ & $>250$ & $P$ value & $>0-125$ & $>125-250$ & $>250$ & $P$ value \\
\hline$N(\%)$ patients & $3211(18.6)$ & $9384(54.3)$ & $4675(27.1)$ & & $2073(13.9)$ & $7754(51.8)$ & $5138(34.3)$ & \\
\hline Age, mean (SD) & $38.2(17.2)$ & $41.2(16.4)$ & $44.3(15.5)$ & & $33.5(18.0)$ & $39.2(16.9)$ & $43.5(15.7)$ & \\
\hline Female sex, $n(\%)$ & $2054(64.0)$ & $5625(59.9)$ & $2716(58.1)$ & & $1245(60.1)$ & $4722(60.9)$ & $3088(60.1)$ & \\
\hline \multicolumn{9}{|c|}{ Severe exacerbations during baseline year, $n$ (\%) } \\
\hline 0 & $2763(86.0)$ & $7766(82.8)$ & $3653(78.1)$ & & $1738(83.8)$ & $6407(82.6)$ & $4103(79.9)$ & \\
\hline 1 & $363(11.3)$ & $1337(14.2)$ & $818(17.5)$ & $<0.001^{*}$ & $270(13.0)$ & $1085(14.0)$ & $794(15.5)$ & $<0.001^{*}$ \\
\hline$>2$ & $85(2.6)$ & $281(3.0)$ & $204(4.4)$ & & $65(3.1)$ & $262(3.4)$ & $241(4.7)$ & \\
\hline
\end{tabular}

$S D$ standard deviation

${ }^{*}$ Chi squared test

${ }^{a}$ Fluticasone propionate equivalent

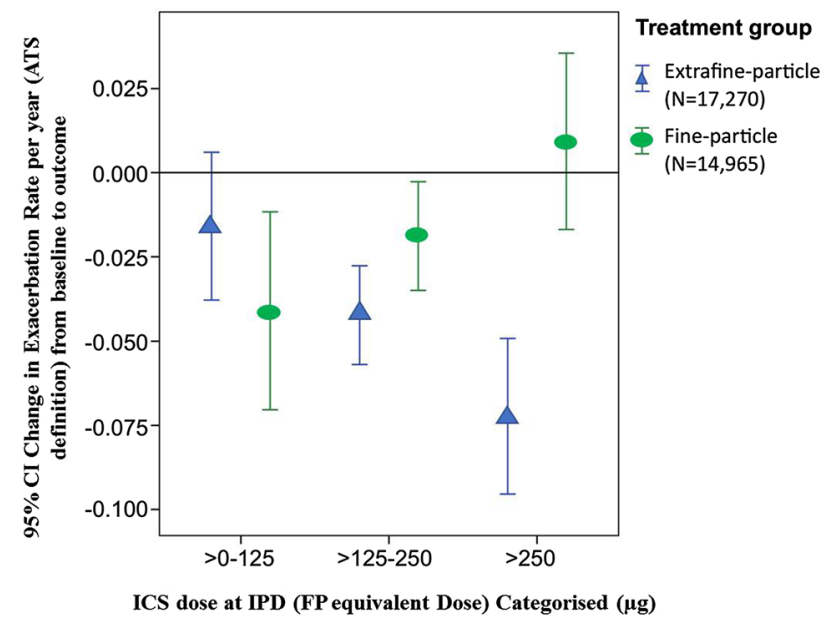

Fig. 1 Mean change in the severe exacerbation rate (ATS/ ERS defined) from baseline to outcome versus ICS dose prescribed at index date for the adult initiation cohort. Note: Exacerbation rate from baseline year to outcome year was compared using the Kruskal-Wallis test. IPD

$[-0.0188(95 \% \mathrm{CI},-0.035,-0.027)]$, but not in the highest dose category [0.009 (95\% CI, $-0.017,0.036)$; Fig. 1, Table 3].

\section{DISCUSSION}

This real-life asthma patient population study found an increasing dose-response relationship
Index Prescription Date, ICS Inhaled Corticosteroids, FP Fluticasone Propionate, $C I$ Confidence Interval, ATS American Thoracic Society, ERS European Respiratory Society

between extrafine ICS and reduction in exacerbation rates in adolescent and adult patients initiated on ICS. However, for patients receiving fine-particle ICS, high doses were not more beneficial than lower doses.

These findings reinforce previous studies in which EF HFA-BDP had a similar or better chance of achieving asthma control than fluticasone $[16,26,27]$. The superior effectiveness of 
Table 3 Outcome characteristics for patients initiated on different doses of extrafine- and fine-particle ICS

\begin{tabular}{|c|c|c|c|c|c|c|c|c|}
\hline \multirow[b]{2}{*}{ Characteristic } & \multicolumn{4}{|c|}{$\begin{array}{l}\text { Extrafine-particle ICS } \\
\text { daily dose at initiation } \\
(\mu \mathrm{g})\end{array}$} & \multicolumn{4}{|c|}{$\begin{array}{l}\text { Fine-particle ICS daily } \\
\text { dose at initiation }^{\mathrm{a}} \\
(\mu \mathrm{g})\end{array}$} \\
\hline & $>0-125$ & $>125-250$ & $>250$ & $P$ value & $>0-125$ & $>125-250$ & $>250$ & $P$ value \\
\hline \multicolumn{9}{|c|}{ Severe exacerbations during outcome year, $n$ (\%) } \\
\hline 0 & $2864(89.2)$ & $8281(88.2)$ & $3986(85.3)$ & & $1807(87.2)$ & $6684(86.2)$ & $4303(83.7)$ & \\
\hline 1 & $253(7.9)$ & $804(8.6)$ & $499(10.7)$ & $<0.001^{*}$ & $209(10.1)$ & $800(10.3)$ & $563(11.0)$ & $<0.001^{*}$ \\
\hline$>2$ & $94(2.9)$ & $299(3.2)$ & $190(4.1)$ & & $57(2.7)$ & $270(3.5)$ & $272(5.3)$ & \\
\hline
\end{tabular}

* Chi squared test

${ }^{a}$ Fluticasone propionate equivalent

extrafine-particle ICS observed in this study may be explained by improved airway drug deposition and distribution. The results of the present study add to the evidence in support of the use of extrafine-particle ICS to treat small airway inflammation [26, 28, 29].

A non-randomized, open-label study, using scintigraphic assessment, demonstrated that $50 \%$ of the ex-actuator dose of ciclesonide extrafine particles was deposited in the lungs, and that the highest deposition occurred in the peripheral regions containing the small airways [30]. 3D SPECT imaging revealed that the highest deposition occurred within the peripheral regions of the lung that contain the small airways and alveoli and low oropharyngeal deposition [30]. The authors concluded that this combination may contribute to the reduced incidence of side effects associated with ciclesonide use. Inflammation and patency of small airways was shown to improve with ciclesonide suggesting that it exerts an anti-inflammatory effect on small airways [13]. A real-world pilot study by Hodgson et al. [31] found that, in patients with severe asthma, there was significantly less airway eosinophilia with ciclesonide than with placebo. In addition to improved asthma control, effective targeting of the small airways, at a lower dose, may have the potential to reduce local and systemic side effects [32].

There is increasing evidence to support the hypothesis that small airway inflammation contributes to difficulties in asthma control and that extrafine ICS can control this. This may also explain why the effect of increasing dose of patients initiating on fine-particle ICS is less steep than that seen with the extrafine ICS. Distal lung disease has been associated with a risk of recurrent asthma exacerbations [32]; therefore, small airways may be important in patients with more severe disease. These patients are often those treated with higher doses of ICS. The use of severe exacerbations as the primary outcome in the present study highlights a real-life clinical benefit of improved small airway deposition.

RCTs are generally representative of only fewer than 5\% of all patients with asthma [33] and have limited inclusion criteria, which often exclude typical patients seen in general practice, such as smokers and those with comorbidities, and those that suffer from severe or unstable disease. Study durations are short, ranging from six days to three months. Licensing studies are often designed to show equivalence and often take stable populations. The differences in efficacy between RCTs and real-world studies could be partly due to high adherence in such studies, due to constant monitoring of the patient. Adherence is a prerequisite for enrolment into RCTs to maximize the treatment effects. Patients enrolled in studies tend to pay closer attention to their health and are more likely to follow instructions. This does not reflect the situation in the general patient population where adherence tends to be relatively low [34]. The generalizable nature of these findings has the potential to inform future 
changes in practice and thus have an early clinical impact. Therefore, evidence from realworld studies is an important addition to evidence from RCTs.

As with other observational studies, there is a potential for selection and physician bias and residual confounding. Furthermore, the analysis was based on the prescribed dose at ICS initiation, as opposed to ICS doses during the outcome year. In this observational study the response to consumed dose would not be a meaningful analysis as the results would therefore be confounded by severity. We relied on prescription information from the primary care databases we used. This was an intention-to-treat analysis, thus the data allowed us to identify instances of when prescriptions were written by GPs. It did not include information on whether the prescription was filled, and thus we are unable to guarantee consumption of the medications in this study. However, this does not rule out that the relationship between initial severity and dose response efficacy is attributable to decreased responsiveness at lower doses among patients with more severe asthma [35], rather than to increased responsiveness to medication. Study findings are dependent on the availability and quality of the data in the OPCRD and CPRD, which contain limited information on hospitalizations.

At the time of this study both ciclesonide and EF HFA-BDP were prescribed for treatment of asthma in the UK. Although we did not specifically look at how many of the patients examined were on different EF ICS preparations, most patients in the UK, at the time of this study, prescribed extrafine ICS were given beclomethasone, not ciclesonide. A recent publication has shown that ciclesonide and $\mathrm{EF}$ HFA-BDP have similar potency and that oncedaily dosing of beclomethasone and ciclesonide is similar in efficacy [36]. It is therefore unlikely for individual drug type to have a significant effect on the outcomes.

A measure of exhaled nitric oxide would allow a more concrete evaluation of the effect of treatment on the peripheral airways, as this has previously been reported to decrease upon use of EF-BDP compared with the non-extrafine formulation [37]. Nonetheless, the OPCRD is accepted as a high-quality database with the advantages of large size and inclusion of a representative sample of the UK population. The use of patient-centered severe exacerbations as the primary outcome further strengthens this study. Evaluation over one full baseline year and one full outcome year was done to ensure minimal effect of seasonal variations as well as ensure recording of any infrequent outcomes, such as exacerbations. The inclusion and exclusion criteria were selected to minimize potential confounding factors such as other asthma pharmacotherapies, and to identify patients from a large clinical population receiving initial ICS therapy.

\section{CONCLUSIONS}

In a real-life population of adult patients with asthma initiating ICS therapy, patients starting with higher doses of extrafine-particle size ICS had a greater reduction in severe exacerbation rates, compared with those initiating on low or medium doses. This dose-response effect was not observed with initiation of fine-particle ICS. This supports the hypothesis that targeting the small airways, using extrafine-particle ICS, may improve the dose-response to inhaled corticosteroids in asthma. Further prospective observational studies and pragmatic trials are needed to confirm this hypothesis.

\section{ACKNOWLEDGEMENTS}

This study was conducted in collaboration with Teva Pharmaceuticals Europe B.V., who also provided full funding support for the study and article processing charges. All named authors meet the International Committee of Medical Journal Editors (ICMJE) criteria for authorship for this manuscript, take responsibility for the integrity of the work and have given final approval to the version to be published. All authors had full access to all of the data in this study and take complete responsibility for the integrity of the data and accuracy of the data analysis. The authors would like to thank Martina Stagno d'Alcontres, a current employee of 
Observational and Pragmatic Research Institute (OPRI), and Rosalind Bonomally, a former employee of OPRI, for medical writing. Medical writing/editorial assistance was funded by OPRI. Many thanks to Derek Skinner, current employee of Optimum Patient Care, for preparation of data for analysis.

Disclosures. The University of Groningen has received money for Dirkje S. Postma regarding an unrestricted educational grant for research from Astra Zeneca, Chiesi. Travel to ERS and/or ATS has been partially funded by AstraZeneca, Chiesi, GSK, Takeda. Fees for consultancies were given to the University of Groningen by AstraZeneca, Boehringer Ingelheim, Chiesi, GSK, Takeda, and TEVA. Travel and lectures in China paid by Chiesi. Alan Kaplan is either on the advisory board or speakers' bureau for Astra Zeneca, Boehringer Ingelheim, Griffols, GSK, Johnson and Johnson, Meda, Merck Frosst, Novartis, Pfizer, Purdue and Teva. Joan B. Soriano was employed by Observational and Pragmatic Research Institute Pte Ltd, at the time of the study. He has received pharmaceutical company grants from GSK in 2011 and Chiesi in 2012 via CIMERA, his former home institution, and in 2014 and 2015 from Linde via Hospital Universitario de La Princesa; he participated in speaking activities, advisory committees and consultancies during the period 2011-2016 sponsored by: Almirall, AstraZeneca, Boehringer-Ingelheim, Chiesi, ERS, GEBRO, Grifols, GSK, Linde, Lipopharma, Mundipharma, Novartis, Pfizer, OPRI, Rovi, SEPAR, Takeda, and Teva. Jonathon Grigg received honoraria from Novartis as a member of an advisory board for an asthma medication and received travel expenses and hotel accommodation from Observational and Pragmatic Research Institute Pte Ltd, to attend meetings related to the study. Theresa W. Guilbert reports personal fees from American Board of Pediatrics; Pediatric Pulmonary Subboard, grants and personal fees from GSK, personal fees from Regeneron Pharmaceuticals, grants from $\mathrm{NIH}$, other from UpToDate, personal fees from Merck, personal fees and other from Sanofi, personal fees and other from Novartis, outside the submitted work. Wim van Aalderen is a member of Medical Advisory Board of AstraZeneca. Nicolas Roche has received over the past 3 years (i) fees for speaking, organizing education, participation in advisory boards or consulting from $3 \mathrm{M}$, Aerocrine, Almirall, AstraZeneca, Boehringer Ingelheim, Chiesi, Cipla, GlaxoSmithKline, MSD-Chibret, Mundipharma, Novartis, Pfizer, Sanofi, Sandoz, Teva; (ii) research grants from Novartis, Boehringer Ingelheim and Pfizer. Anne Burden was an employee of OPRI, which conducted this study and which has conducted paid research in respiratory disease on behalf of the following other organizations in the past 5 years: Aerocrine, AKL Research and Development Ltd, Almirall, AstraZeneca, Boehringer Ingelheim, Chiesi, GlaxoSmithKline, Meda, Mundipharma, Napp, Novartis, Orion, Takeda, Teva, and Zentiva. Elizabeth V. Hillyer is a consultant to the OPRI and has received payment for writing and editorial support to Merck. Elliot Israel reports receiving Consulting fees from AstraZeneca, Bird Rock Bio, Cowen \&amp; Co, Novartis, Nuvelution Pharmaceutical, Philips Respironics, Regeneron Pharmaceuticals, TEVA Specialty Pharmaceuticals and Vitaeris, Inc; fees for Expert Testimony from Campbell, Campbell, Edwards \&Conroy, Crammer, Bishop \& O’Brien, Fox Rothschild, and Ryan Deluca LLP; Travel Grant Support from Research in Real Life (RiRL), TEVA Specialty Pharmaceuticals; Royalties from UpToDate, Deputy Editor Fees from the American Thoracic Society, DSMB Member for Novartis with No Compensation, and having Grant Support paid to his Institution from Genentech, Sanofi, and the NIH. David B. Price has board membership with Aerocrine, Amgen, AstraZeneca, Boehringer Ingelheim, Chiesi, Meda, Mundipharma, Napp, Novartis, and Teva Pharmaceuticals; consultancy with Almirall, Amgen, AstraZeneca, Boehringer Ingelheim, Chiesi, GlaxoSmithKline, Meda, Mundipharma, Napp, Novartis, Pfizer, Teva Pharmaceuticals, and Theravance; grants and unrestricted funding for investigator-initiated studies (conducted through Observational and Pragmatic Research Institute Pte Ltd) from UK National Health Service, British Lung Foundation, Aerocrine, AKL Research and Development Ltd, AstraZeneca, Boehringer Ingelheim, Chiesi, Meda, 
Mundipharma, Napp, Novartis, Pfizer, Respiratory Effectiveness Group, Takeda, Teva Pharmaceuticals, Zentiva, and Theravance; payment for lectures/speaking engagements from Almirall, AstraZeneca, Boehringer Ingelheim, Chiesi, Cipla, GlaxoSmithKline, Kyorin, Meda, Merck, Mundipharma, Novartis, Pfizer, Skyepharma, Takeda, and Teva Pharmaceuticals; payment for manuscript preparation from Mundipharma and Teva Pharmaceuticals; payment for the development of educational materials from Novartis and Mundipharma; payment for travel/accommodation/meeting expenses from Aerocrine, Boehringer Ingelheim, Mundipharma, Napp, Novartis, Teva Pharmaceuticals, and AstraZeneca; funding for patient enrolment or completion of research from Chiesi, Teva Pharmaceuticals, Zentiva, and Novartis; stock/stock options from AKL Research and Development Ltd which produces phytopharmaceuticals; owns $74 \%$ of the social enterprise Optimum Patient Care Ltd, UK and $74 \%$ of Observational and Pragmatic Research Institute Pte Ltd, Singapore; and is peer reviewer for grant committees of the Medical Research Council, Efficacy and Mechanism Evaluation Programme, and Health Technology Assessment.

Compliance with Ethics Guidelines. This was a retrospective study utilizing anonymized patient data, for which formal consent is not required.

Data Availability. The datasets analyzed during the current study are available from the corresponding author on reasonable request.

Funding. Teva Pharmaceuticals Europe B.V.

Open Access. This article is distributed under the terms of the Creative Commons Attribution-NonCommercial 4.0 International License (http://creativecommons.org/licenses/ by-nc/4.0/), which permits any noncommercial use, distribution, and reproduction in any medium, provided you give appropriate credit to the original author(s) and the source, provide a link to the Creative Commons license, and indicate if changes were made.

\section{REFERENCES}

1. van der Wiel E, ten Hacken NH, Postma DS, van den Berge M. Small-airways dysfunction associates with respiratory symptoms and clinical features of asthma: a systematic review. J Allergy Clin Immunol. 2013;131(3):646-57.

2. Dolhnikoff M, da Silva LF, de Araujo BB, Gomes HA, Fernezlian S, Mulder A, et al. The outer wall of small airways is a major site of remodeling in fatal asthma. J Allergy Clin Immunol. 2009;123(5):1090-7.

3. Burgel PR, de Blic J, Chanez P, Delacourt C, Devillier $\mathrm{P}$, Didier A, et al. Update on the roles of distal airways in asthma. Eur Respir Rev. 2009;18(112):80-95.

4. From the Global strategy for asthma management and prevention, global initiative for asthma. 2016 . http://ginasthma.org/. Accessed 7 Apr 2016.

5. Powell H, Gibson PG. Inhaled corticosteroid doses in asthma: an evidence-based approach. Med J Aust. $2003 ; 178(5): 223-5$.

6. Holt S, Suder A, Weatherall M, Cheng S, Shirtcliffe P, Beasley R. Dose-response relation of inhaled fluticasone propionate in adolescents and adults with asthma: meta-analysis. BMJ. 2001;323(7307):253-6.

7. Masoli M, Weatherall M, Holt S, Beasley R. Clinical dose-response relationship of fluticasone propionate in adults with asthma. Thorax. 2004;59(1):16-20.

8. Baraket M, Oliver BG, Burgess JK, Lim S, King GG, Black JL. Is low dose inhaled corticosteroid therapy as effective for inflammation and remodeling in asthma? A randomized, parallel group study. Respir Res. 2012;13:11.

9. van den Berge M, ten Hacken NH, Cohen J, Douma WR, Postma DS. Small airway disease in asthma and COPD: clinical implications. Chest. 2011;139(2):412-23.

10. Derendorf $\mathrm{H}$, Nave R, Drollmann A, Cerasoli F, Wurst W. Relevance of pharmacokinetics and pharmacodynamics of inhaled corticosteroids to asthma. Eur Respir J. 2006;28(5):1042-50.

11. Bjermer L. History and future perspectives of treating asthma as a systemic and small airways disease. Respir Med. 2001;95(9):703-19.

12. Vanden Burgt JA, Busse WW, Martin RJ, Szefler SJ, Donnell D. Efficacy and safety overview of a new 
inhaled corticosteroid, QVAR (hydrofluoroalkane-beclomethasone extrafine inhalation aerosol), in asthma. J Allergy Clin Immunol. 2000;106(6):1209-26.

13. Cohen J, Douma WR, ten Hacken NH, Vonk JM, Oudkerk M, Postma DS. Ciclesonide improves measures of small airway involvement in asthma. Eur Respir J. 2008;31(6):1213-20.

14. Leach CL, Kuehl PJ, Chand R, McDonald JD. Respiratory tract deposition of HFA-beclomethasone and HFA-fluticasone in asthmatic patients. J Aerosol Med Pulm Drug Deliv. 2016;29(2):127-33.

15. Colice G, Martin RJ, Israel E, Roche N, Barnes $\mathrm{N}$, Burden A, et al. Asthma outcomes and costs of therapy with extrafine beclomethasone and fluticasone. J Allergy Clin Immunol. 2013;132(1):45-54.

16. Price D, Martin RJ, Barnes N, Dorinsky P, Israel E, Roche $\mathrm{N}$, et al. Prescribing practices and asthma control with hydrofluoroalkane-beclomethasone and fluticasone: a real-world observational study. J Allergy Clin Immunol. 2010;126(3):511.

17. Krishnan JA, Schatz M, Apter AJ. A call for action: comparative effectiveness research in asthma. J Allergy Clin Immunol. 2011;127(1):123-7.

18. Price D, Chisholm A, van der Molen $\mathrm{T}$, Roche $\mathrm{N}$, Hillyer EV, Bousquet J. Reassessing the evidence hierarchy in asthma: evaluating comparative effectiveness. Curr Allergy Asthma Rep. 2011;11(6):526-38.

19. Rawlins M. De testimonio: on the evidence for decisions about the use of therapeutic interventions. Lancet. 2008;372(9656):2152-61.

20. OPCRD. The Optimum Patient Care Research Database (OPCRD) $2016 \quad$ [http:// optimumpatientcare.org/opcrd/]. Accessed 21 Dec 2016.

21. CPRD. The Clinical Practice Research Datalink (CPRD) (updated 16 Dec 2015).

22. Williams T, van Staa T, Puri S, Eaton S. Recent advances in the utility and use of the General Practice Research Database as an example of a UK Primary Care Data resource. Ther Adv Drug Saf. 2012;3(2):89-99.

23. Tate AR, Beloff N, Al-Radwan B, Wickson J, Puri S, Williams T, et al. Exploiting the potential of large databases of electronic health records for research using rapid search algorithms and an intuitive query interface. $\mathrm{J}$ Am Med Inform Assoc. 2014;21(2):292-8.
24. Reddel HK, Taylor DR, Bateman ED, Boulet LP, Boushey HA, Busse WW, et al. An official American Thoracic Society/European Respiratory Society statement: asthma control and exacerbations: standardizing endpoints for clinical asthma trials and clinical practice. Am J Respir Crit Care Med. 2009;180(1):59-99.

25. Global Initiative on Asthma (GINA). Global strategy for asthma management and prevention 2016. http://ginasthma.org/. Accessed 7 Apr 2016.

26. Busse WW, Brazinsky S, Jacobson K, Stricker W, Schmitt K, Vanden Burgt J, et al. Efficacy response of inhaled beclomethasone dipropionate in asthma is proportional to dose and is improved by formulation with a new propellant. J Allergy Clin Immunol. 1999;104(6):1215-22.

27. Turner S, Richardson K, Murray C, Thomas M, Hillyer EV, Burden A, et al. Long-acting beta-agonist in combination or separate inhaler as step-up therapy for children with uncontrolled asthma receiving inhaled corticosteroids. J Allergy Clin Immunol Pract. 2017;5(1):99-106.

28. Leach CL, Davidson PJ, Hasselquist BE, Boudreau RJ. Lung deposition of hydrofluoroalkane-134a beclomethasone is greater than that of chlorofluorocarbon fluticasone and chlorofluorocarbon beclomethasone: a cross-over study in healthy volunteers. Chest. 2002;122(2):510-6.

29. Thongngarm T, Silkoff PE, Kossack WS, Nelson HS. Hydrofluoroalkane-134A beclomethasone or chlorofluorocarbon fluticasone: effect on small airways in poorly controlled asthma. J Asthma. 2005;42(4):257-63.

30. Newman S, Salmon A, Nave R, Drollmann A. High lung deposition of $99 \mathrm{mTc}$-labeled ciclesonide administered via HFA-MDI to patients with asthma. Respir Med. 2006;100(3):375-84.

31. Hodgson D, Anderson J, Reynolds C, Meakin G, Bailey $\mathrm{H}$, Pavord I, et al. A randomised controlled trial of small particle inhaled steroids in refractory eosinophilic asthma (SPIRA). Thorax. 2015;70(6):559-65.

32. Martin RJ. Therapeutic significance of distal airway inflammation in asthma. J Allergy Clin Immunol. 2002;109(2 Suppl):S447-60.

33. Herland K, Akselsen JP, Skjonsberg OH, Bjermer L. How representative are clinical study patients with asthma or COPD for a larger "real life" population of patients with obstructive lung disease? Respir Med. 2005;99(1):11-9. 
34. Saturni S, Bellini F, Braido F, Paggiaro P, Sanduzzi A, Scichilone $\mathrm{N}$, et al. Randomized controlled trials and real life studies. Approaches and methodologies: a clinical point of view. Pulm Pharmacol Ther. 2014;27(2):129-38.

35. Adams N, Bestall JM, Jones PW. Inhaled beclomethasone versus budesonide for chronic asthma. Cochrane Database Syst Rev. 2002;1:CD003530.
36. Daley-Yates PT. Inhaled corticosteroids: potency, dose equivalence and therapeutic index. Br J Clin Pharmacol. 2015;80(3):372-80.

37. Nicolini G, Chetta A, Simonazzi A, Tzani P, Aiello $\mathrm{M}$, Olivieri D. Both bronchial and alveolar exhaled nitric oxide are reduced with extrafine beclomethasone dipropionate in asthma. Allergy Asthma Proc. 2010;31(5):85-90. 